\title{
The Global Spine Care Initiative: care pathway for people with spine-related concerns
}

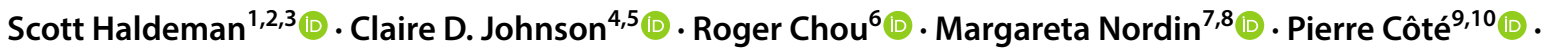

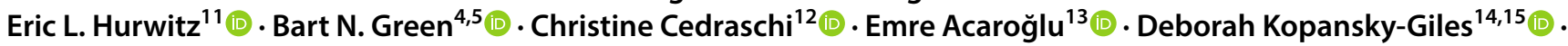 \\ Arthur Ameis $^{16}$ - Afua Adjei-Kwayisi ${ }^{17}$ (1) . Selim Ayhan ${ }^{18}$ (D) Fiona Blyth ${ }^{19} \cdot$ David Borenstein $^{20}$ (1) \\ $\mathrm{O}^{\prime}$ Dane Brady ${ }^{21}$. Peter Brooks ${ }^{22}$. Connie Camilleri ${ }^{23}$. Juan M. Castellote ${ }^{24}$. Michael B. Clay ${ }^{25}$. \\ Fereydoun Davatchi ${ }^{26}$ (1) Robert Dunn ${ }^{27}$ (1) Christine Goertz ${ }^{28,29}$ (E) Erin A. Griffith ${ }^{30}$. Maria Hondras ${ }^{31}$ (1) . \\ Edward J. Kane ${ }^{32}\left(\right.$ Nadège Lemeunier $^{33}$. John Mayer ${ }^{34} \cdot$ Tiro Mmopelwa $^{35}$ - Michael Modic ${ }^{36}$ (D) Jean Moss ${ }^{37}$. \\ Rajani Mullerpatan ${ }^{38}$. Elijah Muteti ${ }^{39} \cdot$ Lillian Mwaniki $^{40} \cdot$ Madeleine Ngandeu-Singwe $^{41} \cdot$ Geoff Outerbridge $^{42}(1)$.

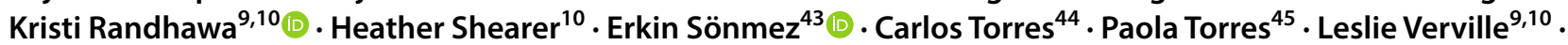 \\ Adriaan Vlok ${ }^{46}$. William Watters $\mathrm{III}^{47,48}$. Chung Chek Wong ${ }^{49} \cdot$ Hainan $\mathrm{Yu}^{9,10}$
}

Received: 12 April 2018 / Accepted: 6 August 2018 / Published online: 27 August 2018

(C) The Author(s) 2018

\begin{abstract}
Purpose The purpose of this report is to describe the development of an evidence-based care pathway that can be implemented globally.

Methods The Global Spine Care Initiative (GSCI) care pathway development team extracted interventions recommended for the management of spinal disorders from six GSCI articles that synthesized the available evidence from guidelines and relevant literature. Sixty-eight international and interprofessional clinicians and scientists with expertise in spine-related conditions were invited to participate. An iterative consensus process was used.

Results After three rounds of review, 46 experts from 16 countries reached consensus for the care pathway that includes five decision steps: awareness, initial triage, provider assessment, interventions (e.g., non-invasive treatment; invasive treatment; psychological and social intervention; prevention and public health; specialty care and interprofessional management), and outcomes. The care pathway can be used to guide the management of patients with any spine-related concern (e.g., back and neck pain, deformity, spinal injury, neurological conditions, pathology, spinal diseases). The pathway is simple and can be incorporated into educational tools, decision-making trees, and electronic medical records.

Conclusion A care pathway for the management of individuals presenting with spine-related concerns includes evidencebased recommendations to guide health care providers in the management of common spinal disorders. The proposed pathway is person-centered and evidence-based. The acceptability and utility of this care pathway will need to be evaluated in various communities, especially in low- and middle-income countries, with different cultural background and resources.
\end{abstract}

Electronic supplementary material The online version of this article (https://doi.org/10.1007/s00586-018-5721-y) contains supplementary material, which is available to authorized users.

Extended author information available on the last page of the article 
Graphical abstract These slides can be retrieved under Electronic Supplementary Material.
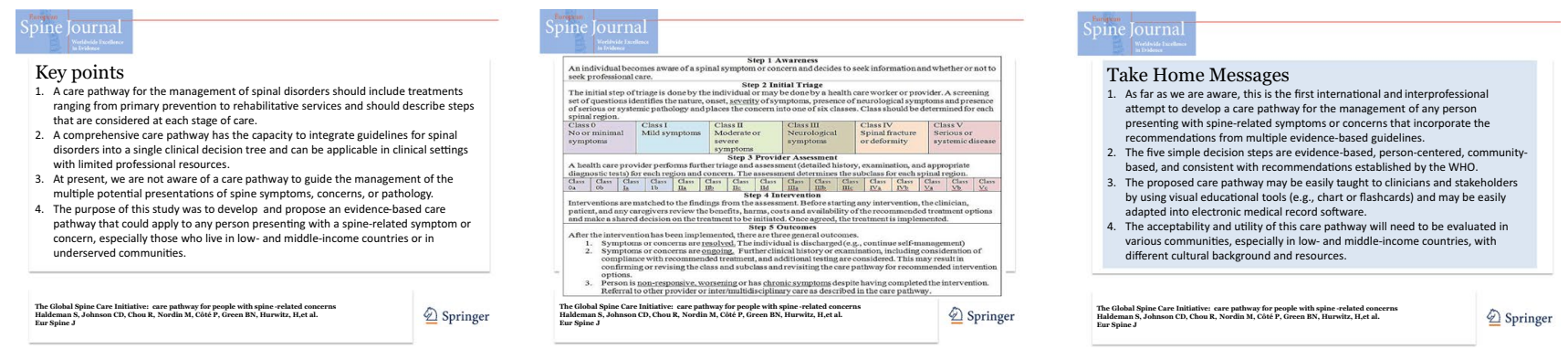

Keywords Delivery of health care $\cdot$ Triage $\cdot$ Evidence-based practice $\cdot$ Quality of health care

\section{Introduction}

In the last two decades, there has been a significant increase in the burden and costs of spinal disorders throughout the world. This impact has been seen not only in high-income countries but also in low- and middleincome countries [1,2]. Non-communicable diseases such as spinal disorders are contributing a higher proportion of the total burden of disease than previously considered. At the same time in low- and middle-income countries, minimal health care resources are being expended internally and through international health care priorities to reduce the disability associated with spinal disorders. In high-income countries, such as the USA, there has been a marked and progressive increase in the cost of health care for people with spinal disorders with a concomitant increase in spine-related disability [3, 4].

Disability and costs attributed to spinal pain are projected to increase, which is a concern particularly for lowand middle-income countries where the infrastructure may be fragile and not equipped to cope with a growing burden [5]. There is an increasing call to action by leaders in the spine care community who stress it is essential to prevent the use of practices that are ineffective, harmful or wasteful of limited resources [6]. The concern is that fragmented and outdated models of care fail to address widespread misconceptions in the population and among health professionals about the causes, prognosis, and effectiveness of different treatments for spinal pain [6].

Controlled clinical trials have been the primary tool to assess the benefits and harms of common interventions for the management of spinal pain with the assumption that this will improve outcomes and reduce the costs and disability associated with spinal disorders. These trials have been performed in high-income countries, so little is known about low- and middle-income countries. At the same time, task forces, government, and professional committees have reviewed and synthesized the results of this research to develop clinical practice guidelines. These guidelines have focused primarily on non-specific or axial low back and neck pain although guidelines have also been developed to address specific pathological spinal disorders, such as spinal stenosis, spondyloarthropathies, scoliosis, and radiculopathy. Implementation of these guidelines, however, has proved difficult [7-9]. The difficulty in implementation and failure of clinicians to follow guidelines in high-income countries has been attributed to complex issues including provider and patient behavior, health literacy, legal, and systems/reimbursement issues.

There are challenges to implementing evidence-based guidelines in any health care community. In many communities, health care providers are scarce [10] or the primary care clinician or team of providers may not have the experience, relevant professional training, or resources to implement evidence-based management for the wide spectrum of spinal disorders. For low- and middle-income countries, resources are scarce $[11,12]$, thus providers may be less likely to have the skills and resources to manage spine-related concerns. Patients in these settings present with a full spectrum of spine-related disorders such as low back, middle back and neck pain, which might be acute, chronic, and/or episodic or recurrent and associated with varying degrees of impairment of function or activities of daily living. The incidence of chronic diseases in low- and middle-income countries is higher than in high-income countries [13, 14]. As well, primary health care could perform better within the care delivery system in underserved areas $[12,15]$. There may be no imaging or laboratory testing facilities readily available at the primary care level and advanced imaging and testing may not be available even at secondary care centers or district hospitals [16]. Some communities have no or few clinicians that commonly manage spinal disorders, such as chiropractors, psychologists or physical therapists. There may be limited access to medical specialists in the fields of 
psychiatry, rheumatology, neurology, psychiatry, or spine surgery at secondary care and even at the tertiary centers where patients with the most serious spinal pathology are likely to be referred. There may not be resources available to manage the complications of the interventions recommended in guidelines such as gastric bleeding from nonsteroidal anti-inflammatory drugs (NSAIDs), constipation, and addiction from opioids, follow-up care and rehabilitation following surgery and psychiatric and psychological care that often is associated with chronic pain. Thus, there is a need to develop a care pathway that could address these concerns and that could be adapted to the community and available resources.

Care pathways are "a complex intervention for mutual (between patient and clinician) decision-making and organization of care processes for a well-defined group of patients during a well-defined period" [17]. Care pathways have been proposed as a means of implementing guidelines into specific clinical settings. A care pathway provides a mechanism whereby multiple guidelines can be integrated and modified to adapt to the available professional and physical resources. They are a means of coordinating health care and to provide a mechanism for integrating evidence-based health care with real-world clinical practice. Chawla et al. [18] describe this process as having the "goal of using high-quality evidence for pathway development and affording physician flexibility in implementation". Care pathways "are used to reduce variation (in care), improve the quality of care, and maximize the outcomes for specific groups of patients" [19]. Cavlan et al. [20] describe care pathways as being organized by the stages of care and including the full range of interventions that may be offered at each stage. They note that pathways should include all treatments, ranging from primary prevention to rehabilitative services, that may be offered to patients and that care pathways should describe steps that are considered at each stage of care. Whereas most current spine-related guidelines tend to address specific subgroups of patients such as non-specific back or neck pain or pathologies such as stenosis or radiculopathy, a comprehensive care pathway has the capacity to integrate guidelines for multiple spinal disorders into a single clinical decision tree and is, therefore, more applicable in clinical settings with limited professional resources.

There is an increased interest in developing and evaluating care pathways for musculoskeletal conditions including low back pain $[21,22]$. However, we are not aware of a care pathway to guide the management of the full spectrum of potential presentations of spinal symptoms, concerns or pathology. This variety includes the most benign questions requiring a public health message through the most severe systemic and life-threatening destructive spinal pathologies while focusing on the most common variations of spinal pain irrespective of location (i.e., neck, middle back, low back, arm, or leg symptoms). However, other care pathways may focus on a particular symptom, such as back pain, or a specific disorder or disease, resulting in a more narrow approach to a patient care pathway. The purpose of this study was to develop a care pathway supported by current evidencebased guidelines that could apply to any person presenting with a spine-related concern, especially those who live in low- and middle-income communities or in underserved communities in high-income countries. At the same time, it was felt to be essential to prevent the use of practices that are ineffective, harmful or wasteful of limited resources and to satisfy reported concerns that fragmented and outdated models of care fail to address widespread misconceptions in the population and among health professionals about the causes, prognosis, and effectiveness of different treatments for spinal pain [6].

\section{Methods}

\section{Criteria for care pathway development}

The Global Spine Care Initiative (GSCI) steering committee, primary authors for the GSCI component articles and guest participants held several meetings to determine the essential characteristics that were required while building the pathway [23]. Criteria used to build the spinal disorders care pathway were selected from the current literature, WHO principles, and from a consensus of panelist expertise. (see Online Resource Figure 1 for guiding principles for the GSCI Pathway). This project was approved by National University of Health Sciences Institutional Review Board (\#H-1503). All authors were informed about the nature of the study and the modified Delphi process and gave written consent by completing an electronic questionnaire.

The following 4 steps were completed to create a care pathway:

Step 1 Determine the broad categories of interventions for spinal disorders

During five face-to-face meetings, the GSCI members used a consensus process to identify six major intervention categories recommended by current guidelines for the management of different categories of spinal disorders: (1) assessment; (2) non-invasive treatment; (3) invasive treatment; (4) psychological and social; (5) prevention and public health; and (6) specialty care and interdisciplinary/multidisciplinary management [24-29]. A review of the literature on compression fractures was included as a case study for spinal symptoms related to systemic pathology, in part because many patients with compression fractures can be managed 
in a primary spine care setting. The literature on the management of other serious or systemic disease with spine-related symptoms (including the inflammatory spondyloarthropathies, infections and neoplastic disorders) was not reviewed due to lack of resources. The description of interventions for these disorders was defined by referral to a specific medical specialist or tertiary spine center.

Step 2 Grouping of interventions into subcategories for the care pathway

Interventions recommended in the reviewed evidence-based guidelines were used to develop the care pathway. An extraction table was created to include the interventions from the GSCI foundational papers. These were consolidated into subcategories that: (1) had similar broad definitions (e.g., reassurance, imaging, non-opioid analgesics, exercise, decompression surgery); (2) could easily be differentiated based on skills or resources (e.g., manual therapy, complex fusion, cognitive behavioral therapy); (3) and had similar evidence of effectiveness and harms (e.g., exercise, non-opioid analgesics, opioids). The extraction table was reviewed by the primary authors for each of the six review articles, the principal investigators, GSCI scientific secretariat, and other GSCI co-authors to ensure that the reported content was compatible with the evidence and met reasonable health care principles. Where there was disagreement between authors, the final decision to include an intervention was determined by the primary authors of the six articles in which the evidence had been reviewed.

Step 3 Link interventions to the GSCI Spinal Disorders Classification system

Content from the intervention categories was linked with the different presentations as defined by the GSCI Classification system [30]. The resulting tables were reviewed by the GSCI executive team and intervention paper authors for agreement. Once an agreement was achieved, the tables were circulated among other members of the GSCI for comment and discussion.

Step 4 Develop a care pathway for people or patients with symptoms or concerns about spinal disorders

The primary authors of the care pathway $(\mathrm{SH}, \mathrm{CJ})$ developed a seed draft of a care pathway utilizing the tables developed in Step 3. The care pathway was based on the principles outlined (see Online Resource Figure 1). Three rounds of review and revision of the care pathway and the article were developed after receiving input from other members of the GSCI executive team and intervention chapter authors. Finally, input was requested from 70 spine care clinicians and researchers that included co-authors of the GSCI articles and individuals selected to ensure that there was both interprofessional and international representation. Responses were obtained from 53, six had no comments and felt that they had not contributed to process, four appeared to have concerns which precluded them serving as co-authors. Therefore, 46 participants from 16 countries (Australia, Botswana, Cameroon, Canada, Chile, France, Ghana, India, Iran, Kenya, Malaysia, South Africa, Spain, Switzerland, Turkey, and USA) provided input and agreed to serve as co-authors of the Care Pathway.

\section{Results}

Figure 1 lists the major assessment; non-invasive treatment; invasive treatment; psychological and social; prevention and public health; specialty care and interprofessional management interventions that were extracted from the reviews of the literature and divided into the six broad intervention categories described in the methodology [24-29, 31, 32]. Under each broad category of intervention, the individual interventions were divided into subsections where the mechanisms of action, outcomes, costs, and skills necessary to provide the service are similar. Interventions from the GSCI review articles are included in this table.

\section{Care pathway}

The GSCI care pathway includes five steps that are common to most clinical decision trees [22]. The GSCI care pathway guides the person who has spine-related symptoms or concerns and the clinician who is consulted through five steps and four decisions that make up the care pathway in Fig. 2. The following is a description of each step.

\section{Step 1 Awareness}

The first step is when an individual with a spine-related symptom or concern becomes aware (i.e., the person has a worry that something is or will be wrong with the spine) and seeks information. The person may request information from friends, family, or health care personnel, or perform a search of the media (e.g., internet, magazines, or professional health care information sources). At this stage of the care pathway, educational materials and public health messages about spine-related concerns should include evidence-based information about risks and prognostic factors, prevention, self-assessment, and self-management. Educational messages and programs should provide adequate information so that the person is informed about when to consider self-care and when to seek care from health care personnel. 


\section{Assessment}

- Clinical history: Detailed clinical history of spine-related symptoms and/or concerns, which includes but is not limited to, location, severity, onset, chronicity, functional ability and other relevant information. General health history of other diseases or symptoms and systems review.

- Clinical examination: Detailed spinal/orthopedic examination and neurological examination of spine-related symptoms and/or concerns. General physical examination

- $\quad$ Assess for red flags: Screen for serious disease with spinal symptoms, signs or symptoms of serious or systemic disease, neurological signs or symptoms likely to result in a permanent neurological loss.

- Diagnostic Imaging: Diagnostic imaging to determine and define structural integrity or pathology. Conventional radiography (x-ray), computed tomography (+/- contrast), magnetic resonance imaging (+/- contrast), diagnostic ultrasound, or bone scan. Imaging should only be ordered with the appropriate indications.

- $\quad$ Laboratory: Laboratory studies to rule in or rule out pathology. These may include blood serology, urinalysis, and screening for inflammatory joint disease or tuberculosis.

\section{Non-Invasive treatment}

- $\quad$ Education about spine conditions: Health care provider shares information in a report of findings regarding the spine-related concern or condition, including diagnosis, prognosis, therapies, alternatives, and consent. Depending upon the presentation, the provider may reassure individual about the benign and self-limiting nature of the typical course of spinal pain that has no serious pathology, advise patients to remain active, and provide information about effective self-care options that address the spinal condition or concern.

- $\quad$ Self-care: information about how one can take care of spinal conditions or concerns. Information distributed by from published material, the internet, public health sources, or other communications.

- Therapeutic exercise: Supervised or prescribed and self-directed exercise regimens aimed at spinal pain or a spinal condition.

- Heat/cold therapy: Recommendation of home use of physical agents. For example, heating pads, heat wraps, hot baths, warm gel packs, ice packs.

- $\quad$ Mind-body therapies: Recommendation for self-directed use of one or more of the following: meditation, yoga, biofeedback, tai chi, qigong, relaxation techniques, hypnosis, guided imagery, stress management, or breathing techniques.

- Manual therapy: Provider delivered manipulation, mobilization, massage, and/or soft-tissue therapies.

- $\quad$ Acupuncture: Provider delivered acupuncture, dry needling, or electro-acupuncture/needling.

- $\quad$ Bracing: Provider directed bracing for treatment of acute, stable vertebral fractures (post-trauma and osteoporotic)

- Non-opioid analgesics: Provider directed consumption of non-steroidal anti-inflammatory drugs (NSAIDS), acetaminophen/paracetamol.

- Muscle relaxants: Provider prescribed muscle relaxants.

- $\quad$ Opioid analgesics: Provider prescribed short-term opioids for acute pain with medication-specific education and close monitoring. Only use opioids when other non-invasive therapies for pain relief are inappropriate or have failed. Refer to current evidence-based guideline for proper opioid prescribing information, including dosage, length of use, and addiction risks.

- $\quad$ Anti-depressants: Provider prescribed for psychiatric disorder or chronic pain (i.e., serotonin and norepinephrine reuptake inhibitors.)

- Calcitonin: Provider prescribed calcitonin for treatment of acute osteoporotic compression fracture.

Invasive treatment

- $\quad$ Epidural injections: Provider delivered epidural injections for radicular pain.

- Simple decompression: Provider delivered one or two level discectomy without fusion.

- $\quad$ Simple fusion: Provider delivered one or two level fusion.

- Complex decompression: Provider delivered multilevel decompression with or without fusion

- $\quad$ Complex fusion: Provider delivered multilevel or complex fusion with stabilizing hardware.

- Vertebral augmentation: Provider delivered vertebroplasty and kyphoplasty for compression fractures.

Psychological and social

- $\quad$ Assessment for psychosocial risk factors: Assess for psychosocial factors (flags), such as individual cognitive and behavioral psychosocial factors known to impact prognosis and disability associated with spinal pain; beliefs, appraisals, and judgments; identify unhelpful beliefs about pain; expectations of poor treatment outcome, delayed return to work; emotional responses; pain behavior; avoidance of activities due to expectations of pain and possible re-injury, over-reliance on passive treatments. Other psychosocial factors include: psychiatric symptoms (e.g., clinical depression); perceptions about relationship between work and health (e.g., perception of inability to change work environment, unsupportive workplace), system obstacles that influence psychosocial factors (e.g., legislation, conflict with others, unable to change work duties).

- $\quad$ Reassurance: Provider gives reassurance that the person's concern has been heard. The person is invited to engage in decision-making to address psychosocial factors and is reassured that best care will be provided.

- $\quad$ Psychological and psychosocial therapies: Supervised and/or self-directed therapy should be directed at any relevant psychological and social issues found during assessment. Depending upon the person's needs, this may include approaches such as one of the many forms of cognitive behavioral therapies. For those with chronic pain, provider delivered short-term multidisciplinary programs may address physical limitations and psychosocial barriers to improved function. Behavioral therapy includes education, counseling and functional training with specific activity goals. Self-directed, individual psychological therapies such as meditation and relaxation techniques may be included.

Fig. 1 Categories of evidence-based interventions for spine-related concerns [25-29, 31-33] 


\section{Prevention and public health}

- $\quad$ Assess risk factors and comborbidities: Ask questions during history and use screening tools to identify factors that may worsen present clinical concern or that could relate to future spine-related disorders. Assess for non-modifiable factors (e.g., sex, age, prior history of musculoskeletal disorders, related health conditions); exposures (smoking, risk for unintentional injury/falls, physical job related factors, medications, infections); psychological and social factors (level of education, mental health concerns, job related psychological associations, negative expectations of care); and biological factors (level of physical activity, body mass index, dietary habits, risk for infection). Further evaluate identified risks when indicated with laboratory or diagnostic imaging (e.g., lab tests to screen individuals at risk for tuberculosis, bone densitometry for osteoporosis risk).

- $\quad$ Prevent worsening or occurrence: Match prevention strategy for spine-related disorders with risk factors and comorbidities found during assessment. Educate how to reduce risks and manage comorbidities to prevent the spine-related disorder from occurring (e.g., safe work practices to prevent traumatic spinal injury, fall prevention strategies for the elderly) or decrease future chronicity, severity, and/or disability (e.g., use of occupational safety equipment, those with positive tuberculosis tests should obtain treatment to prevent spinal tuberculosis, smoking cessation to reduce likelihood of back pain becoming chronic, feasible physical activities). Recommend how the individual and care givers may act autonomously to prevent a disorder or decrease future severity and/or disability. Refer or co-manage patients with advanced or comorbid conditions to prevent worsening of spinal disorder (e.g., collabo rate with endocrinologist for a patient with vertebral osteopenia and diabetes).

- $\quad$ Monitor risk factors and comborbidities: Track risk factors and comorbidities and intervene as necessary (e.g., monitor bone density to inform intervention if osteopenia is progressing, monitor body mass index of pregnant women to prevent fetal neural tube defects, reconcile medication list for medications associated with spinal disorders, such as medications for pain, epilepsy, osteoporosis, depression, or gastrointestinal problems.)

- $\quad$ Prevent over-medicalization: Avoid giving patient inaccurate information or advice that is not supported by evidence. Avoid use or promotion of harmful or ineffective procedures in clinic or for self-care. Avoid over-treatment during care. Do not screen for conditions where screening lacks evidence or where benign or self-limiting conditions could be construed as serious.

- $\quad$ Contribute to community health: Collect community and population health information for risk factors and comorbidities (e.g., smoking, overweight, tuberculosis, trauma, level of physical activity). Participate in registries (e.g., contribute to registries for spinal conditions and conditions that influence spine-related disorders). Promote community education programs to prevent locally prevalent spinal disorders and to reduce the community burden of existing disorders. Advocate for community-based interventions and policies.

Specialty care and interdisciplinary/multidisciplinary management

- $\quad$ Multidisciplinary care: "multidisciplinary biopsychosocial rehabilitation programmes involve a combination of physical, psychological, educational, and/or work related components and are often delivered by a team of health care providers with expertise in different fields."(33) This complex type of care is typically reserved for managing chronic pain and is coordinated spinal care that is delivered by a team of providers from multiple disciplines. The multidisciplinary treatment model can be expensive due to the amount of resources necessary to implement. Therefore, preventing chronicity will help avoid the need to use a multidciplinary approach. Team members collaborate together and provide combinations of non-invasive, psychological, rehabilitation and invasive interventions. This type of care is typically reserved for advanced or difficult cases to conserve resources.

- $\quad$ Rheumatology: Refer to appropriate diagnostic and treatment interventions for rheumatoid arthritis and other inflammatory spondyloarthropathies.

- Internal medicine or infectious disease specialist: Refer for appropriated diagnostic and treatment interventions for spinal infections, including discitis, abscess. Surgical intervention when indicated.

- Oncology: Refer for appropriated diagnostic and treatment interventions for appropriate diagnostic and treatment interventions for primary spinal tumors or metastatic spinal lesions.

- Neurology: Refer for appropriated diagnostic and treatment interventions for central pain syndromes, spinal cord injury, plexopathies, muscular dystrophies etc.

- $\quad$ Psychiatry: Refer for appropriated diagnostic and treatment interventions for psychiatric pain syndromes, severe depression associated with spinal pain, symptom magnification.

- $\quad$ Other specialist and subspecialties: Refer for appropriated diagnostic and treatment interventions for specific non-spinal organ diseases with spine-related (referred) symptoms. Refer to a specialist that can manage spinal symptoms likely to originate from internal organ pathology. For example, genito-urinary infection with low back pain (urologist), angina referred to the thoracic spine or upper extremity (cardiologist), arterial dissection or aneurysm with associated spinal pain (vertebral artery dissection causing neck pain, abdominal aneurysm causing low back) (cardiologist, neurologist). Others include internists, obstetrician-gynecologists, general orthopedists and, general surgeon.

- $\quad$ Surgery: Refer to surgeon when indicated (e.g., atlanto-axial ligamentous instability from rheumatoid arthritis).

Fig. 1 (continued)

\section{Step 2 Initial Triage}

After considering information in Step 1, the individual decides whether or not to seek care. Initial triage may be done by those who have adequate education to read and understand the triage questions, thus may include the individual him/herself, friends, family members, health care workers, or health care providers. Information in the care pathway screening questions help to determine which major class of spinal disorder fits the spine-related concern. This information should differentiate between people with nonspecific spinal pain and those who are likely to have more serious pathology requiring more advanced care. 


\section{Step 1 Awareness}

An individual becomes aware of a spinal symptom or concern and decides to seek information and whether or not to seek professional care.

\section{Step 2 Initial Triage}

The initial step of triage is done by the individual or may be done by a health care worker or provider. A screening set of questions identifies the nature, onset, severity of symptoms, presence of neurological symptoms and presence of serious or systemic pathology and places the concern into one of six classes. Class should be determined for each spinal region.

\begin{tabular}{|l|l|l|l|l|l|}
\hline $\begin{array}{l}\text { Class 0 } \\
\text { No or minimal } \\
\text { symptoms }\end{array}$ & $\begin{array}{l}\text { Class I } \\
\text { Mild symptoms }\end{array}$ & $\begin{array}{l}\text { Class II } \\
\text { Moderate or } \\
\text { severe } \\
\text { symptoms }\end{array}$ & $\begin{array}{l}\text { Class III } \\
\text { Neurological } \\
\text { symptoms }\end{array}$ & $\begin{array}{l}\text { Class IV } \\
\text { Spinal fracture } \\
\text { or deformity }\end{array}$ & $\begin{array}{l}\text { Class V } \\
\text { Serious or } \\
\text { systemic disease }\end{array}$ \\
\hline
\end{tabular}

\section{Step 3 Provider Assessment}

A health care provider performs further triage and assessment (detailed history, examination, and appropriate diagnostic tests) for each region and concern. The assessment determines the subclass for each spinal region.

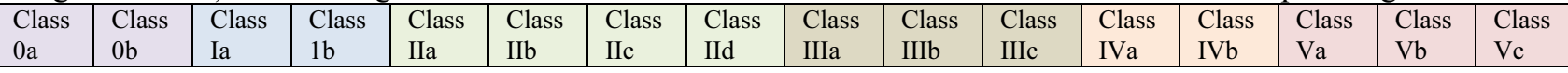

\section{Step 4 Intervention}

Interventions are matched to the findings from the assessment. Before starting any intervention, the clinician, patient, and any caregivers review the benefits, harms, costs and availability of the recommended treatment options and make a shared decision on the treatment to be initiated. Once agreed, the treatment is implemented.

\section{Step 5 Outcomes}

After the intervention has been implemented, there are three general outcomes.

1. Symptoms or concerns are resolved. The individual is discharged (e.g., continue self-management)

2. Symptoms or concerns are ongoing. Further clinical history or examination, including consideration of compliance with recommended treatment, and additional testing are considered. This may result in confirming or revising the class and subclass and revisiting the care pathway for recommended intervention options.

3. Person is non-responsive, worsening or has chronic symptoms despite having completed the intervention. Referral to other provider or inter/multidisciplinary care as described in the care pathway.

Fig. 2 The Global Spine Care Initiative care pathway. A person may have symptoms or concerns in one or more of the following locations: cervical, thoracic, lumbosacral, and/or entire spine. Each region should be considered separately. Reprinted with permission from World Spine Care

Class 0. Triage Screening Questions: (1) Are there any risk factors? (2) Are there any symptoms? (3) If there are symptoms, do they interfere with function or activities? If there are minimal or no spine-related symptoms and the person is looking for information on prevention, prognosis or self-care, he or she may look for information or seek advice from a general health care setting. Examination, further assessment, or interventions beyond advice, education, and prevention recommendations are unlikely to be indicated. The majority of concerns in Class 0 would benefit from information consistent with current guidelines and do not need active care.

Class I. Triage Screening Questions: (1) Is pain acute/ subacute (less than 3 months) or recurrent or chronic (>3 months)? (2) Does pain interfere with function or activities? (3) Are there any neurological deficits? (4) Are there any signs of serious or systemic pathology (red flags)? Class 1 includes non-specific pain in one or more spinal regions that is not interfering with activities and has no neurological symptoms or red flags. Most people in Class I can self-manage their symptoms. If the person is not able to self-manage, the person should seek care for further assistance at a primary care setting ideally from a provider with knowledge and skills in primary spine care.

Class II. Triage Screening Questions: (1) Is pain acute/ subacute (less than 3 months) or recurrent or chronic (> 3 months)? (2) Does pain interfere with function or activities? (3) Are there any neurological deficits? (4) Are there any signs of serious or systemic pathology (red flags)? Class II has non-specific pain in one or more spinal regions that is interfering with function or activities but without neurological symptoms or red flags. The person in this class should seek care in a primary care setting. If more advanced care is needed, the health care provider will refer to the appropriate specialty provider.

Class III. Triage Screening Questions: (1) Are neurological symptoms acute (recent) or chronic (longstanding)? 
(2) Have the neurological symptoms been getting worse? (3) Are there any signs of serious or systemic pathology (red flags)? Class III is when the person has symptoms or signs consistent with neurological compromise (numbness, weakness, loss of balance, gait disturbance or any other symptom or sign that is consistent with a neurological lesion). The person in this class should seek care in a primary care setting as soon as possible especially if the symptoms are progressive or pathologic. If more advanced care is needed, the health care provider will refer to secondary or tertiary care.

Class IV. Triage Screening Questions: (1) Is the deformity stable? (2) Is the deformity acute (recent) or chronic (longstanding)? (3) Are there any symptoms related to the deformity? (4) Are there any signs of serious or systemic pathology (red flags)? Class IV has serious bony structural deformity (e.g., severe scoliosis or congenital bone changes), fracture, dislocation, or instability. The person in this class should seek care in a primary care setting. If symptoms relate to pathology such as acute trauma with the possibility of fracture, severe and progressive scoliosis or instability, the person may require secondary or tertiary care on either emergency or elective basis.

Class V. Triage Screening Questions: (1) Is the condition acute? (2) Is it progressive? (3) Are there any signs of serious or systemic pathology (red flags)? Class V has red flags and spinal symptoms may be associated with potentially serious or systemic pathology. Depending upon the situation, the person may be referred to primary care or may be referred directly to a secondary or tertiary care setting on either emergency or elective basis.

\section{Step 3 Provider assessment}

If the individual triage determines that self-management is insufficient, a health care provider with knowledge and skills in spine care should be sought based upon the answers to the triage questions (e.g., for class I concerns, primary care is appropriate, for Class $\mathrm{V}$ the condition may require emergency care.) Spine assessment should include: (1) a detailed clinical history (e.g., onset, chronicity, severity and impact of the symptoms on function or activities of daily living); (2) the use of assessment tools to measure pain severity, disability, impairment, or other relevant outcomes: (3) identify the presence of psychological and social flags; (4) identify the presence of red flags (serious pathology); (5) relevant spinal, neurological and general physical examination; and (6) appropriate diagnostic imaging or laboratory testing [25, 27-29, 31, 32]. The history, clinical examination, and other assessments provide the information for additional triage and to assign the spine-related concern to a specific class and subclass.

\section{Step 4 Intervention}

Interventions are based upon the previous steps in addition to the assessment findings, available resources, and patient preferences. The clinician engages in a conversation to discuss the benefits, harms, costs, treatment alternatives, availability of the interventions within the clinical setting or community and addresses any concerns or questions the patient may have. Clinicians and patients should consider recent recommendations from the American College of Physicians that recommends non-pharmacological treatments be implemented first [34]. A shared decision is made regarding the most appropriate treatment plan and is agreed upon by the patient/family and the clinician/interdisciplinary team as recommended under WHO principles [35-37] and care pathway principles [17]. The intervention is then initiated with emphasis that the patient must play an active role in his/ her own health decisions.

\section{Step 5 Outcomes}

After the recommended interventions have been administered, completed, or discontinued, the clinician conducts a reassessment to determine the outcome of the intervention. Each patient presentation is unique and may range from benign to life-threatening. Therefore, the frequency and timing of assessments are based on the patient's condition and response to care. Assessments should be decided on using a joint-decision-making process between the patient and health care provider. The follow-up may or may not include additional relevant assessment (e.g., history, examination, or additional testing). In most cases, there are three possible outcomes:

1. If the person has responded favorably, their questions have been answered, and they no longer have a need for further information or other interventions, the person is discharged.

2. If the person has responded somewhat favorably but requests further treatment or information, then a further assessment may be needed to determine whether he or she now falls into the same or less severe class of the GSCI classification system. If another class is assigned, the clinician will follow the steps as outlined above for that class.

3. If the person has not responded favorably in a reasonable timeframe or has noted increased symptoms or disability, then further assessment may be needed to determine whether he or she now falls into the same or more severe class. If in the same class, it is reasonable to consider 
different interventions for the same class and subclass from the table of recommended evidence-based interventions. If the patient has increased symptoms he or she may fall within a different spinal disorders class and require a different set of intervention options or referral to a higher level of spine care. If the patient has tried all available evidence-based interventions for the class and subclass that best describes his or her presentation, then consideration should be given to reassessment of the patient needs to identify other possible underlying reasons for poor response to care that may be outside of the purview of the health care system. Possible additional follow-up actions could include discharging the patient for home-care or referring to a tertiary spine care setting for multidisciplinary care, if these have not been attempted previously.

\section{Considerations}

Several caveats must be considered when implementing the care pathway.

1. Interventions are dependent upon presentation, clinical signs and symptoms, assessment of the evidence, patient preferences, affordability of the interventions, availability and clinician knowledge/skill. Therefore, each individual presentation of a person with a spinerelated concern and the clinical and cultural setting of the presentation should be considered unique. Recommendations should be consistent with the patient presentation and available resources.

2. The evidence and indications for specific interventions are determined from the six GSCI intervention articles and subsequently published evidence-based guidelines. Evidence, however, is not static and, as evidence evolves, the care pathway recommendations should evolve with new knowledge.

3. The evidence for a particular intervention may be stronger for one region of the spine than for other regions. For example, the evidence for interventions of any kind for thoracic pain is very limited. Therefore, the development of treatment options may need extrapolation of the evidence from one spinal region to other spinal regions. This should be consistent with evidence which would suggest that the extrapolation is or is not appropriate.

4. In the absence of red flags that may indicate the presence of a progressive disability or life-threatening diseases, interventions for each class should be viewed as treatment options. This is especially true when benefits, harms, available resources, and costs are similar. Selection of specific interventions should be determined by available resources, clinical knowl- edge, GSCI class of spinal disorder, the training and skill of the clinician providing care and patient preference.

5. Education of the public and individuals should include information on benefits, risk, and costs of all interventions irrespective of the class of spinal disorders.

6. A person may have more than one spinal disorder and therefore may be classified in more than one class (e.g., one class for neck pain and a different class for low back pain). From the provider point of view, the selection of interventions should match the evidencebased recommendations for the class of spinal disorders which may be different for different regions of the spine.

7. Management should be prioritized to the area of the spine that is the greatest of the individual's concern or which requires the most immediate intervention to prevent progression of the condition or further harm to the patient.

8. With some exceptions, spinal disorders are dynamic health conditions. Therefore, their classification and management are also dynamic. An individual can move within and between classes as the condition evolves. Therefore, clinicians should monitor patients and if needed, adjust the classification during the evolution of each patient's symptoms, impairment, or disability. The selected interventions should be consistent with the most current classification of spinal disorders and not necessarily with the initial classification.

9. In the majority of cases of non-specific spinal pain, management can be accomplished by a clinician with the skills and knowledge found within a primary spine care setting and it may not be necessary to consider referral to multiple clinicians or to secondary or tertiary spine care settings [38]. However, if multiple clinicians are involved in the care, at all stages of the care pathway, there should be communication among the team members and with the individual seeking care and his or her family.

10. In the case where there are multiple clinicians involved in care, there must be close coordination of the roles, a common goal for the interventions and sequencing of activities of each member of the multidisciplinary care team to avoid duplication, conflicting or unnecessary delays in services.

11. Spine care should not be considered a vertical or standalone service as this would risk disruption or fragmentation of already under-resourced health systems, especially in low- and middle-income countries. As noted by Green et al [29] and Cedrashi et al [27], spinal symptoms are commonly associated with a wide variety of co-morbid diseases and psychological and social factors that often have to be managed at the same time 
as the spinal symptoms. The current thinking is that initiatives should be integrated into health systems in low- and middle-income countries [39].

\section{Discussion}

This is the first attempt by an interprofessional and international task force to create a care pathway that may accommodate all individuals presenting with spine-related symptoms, concerns, or risk factors. The proposed care pathway is based on evidence-based practices and patient's expectations and allows the clinician a level of flexibility based on available resources $[17,18]$. We hope that this pathway provides a consistent approach to the provision of health care services while at the same time considering available health care resources in underserved communities.

The GSCI Care Pathway is consistent with a proposed approach to low back pain [40]. This approach uses current evidence to guide practice and focuses on the importance of public health policy, self-care and education of the general population, patients and clinicians concerning prevention strategies and expectation from interventions $[5,6]$. It is insufficient to focus only on low back pain. Instead, we must also include neck concerns and other spine-related symptoms and the higher incidence of untreated serious spinal pathology that may present in underserved communities.

We recommend a care pathway with five sequential steps irrespective of the clinical setting. Our hope is that its simplicity may facilitate communication between clinicians, patients, and their communities. The pathway aims to reduce confusion by clarifying the most appropriate manner in which a person with spinal symptoms should be managed. Improved communication allows for interventions that emphasize appropriate self-care and avoids reliance on unnecessary treatments. Our assumption is that common understanding and application of an evidence-based approach to spinal disorders will lead to improved outcomes and less costly care.

The care pathway, however, requires that clinicians who manage people with spine-related symptoms have the knowledge, skills, and resources to apply evidence-based care. The pathway provides a guide to what care should be considered in primary, secondary and tertiary spine care settings [41]. The pathway does not require high cost, high technology interventions for the majority of people who present with spinal concerns. In this way, the care pathway may be implemented in most clinical settings, but especially in communities with limited resources such as in low- and middleincome countries. For primary, secondary and tertiary settings that do not have providers with the knowledge, skills, and resources to apply evidence-based spine care, it may be necessary to establish a training program.
The care pathway may be used by clinicians from different clinical disciplines and different specialties or if a clinician has limited training in managing of spinal disorders. The care pathway was developed so it could be displayed in a chart or easily adapted into an electronic medical record or educational tool. A clinician should be able to review the pathway and quickly determine the class or subclass, the assessments, and the relevant evidence-based interventions. The clinician can then select which recommended interventions are available in the current clinical setting and then review the options with the patient and/or family members to describe the benefits, harms, and costs of the different intervention options and incorporate the patient's preferences. This approach is consistent with the WHO principles of interprofessional "collaborative practice-ready" health care [36]. The care pathway also follows the WHO principles of incorporating integrated, people-centered health services, which is considered an essential element of continuity of care, defragmenting of health systems and improving health care outcomes by the WHO [35-37].

This care pathway was developed to be implemented in communities with varying resource capacities, available health care providers, and public health personnel. The triage process helps to differentiate people who may not require professional spine care from those who can be managed with available resources in a health care setting, and those who require referral to a health care setting with higher levels of resources. Low-income communities may consider prioritizing interventions within the options recommended for each class to conform to the available resources including the relative costs of, and availability of the treatment options.

\section{Strengths and limitations}

The GSCI care pathway was developed by an international, interprofessional team of clinicians, scientists, and stakeholders from high-, middle- and low-income countries and based on a model recommended by the WHO which includes person-centered health services [35-37]. The pathway considers available resources, clinician skills, and patient preferences and focusses on evidence-based interventions. It is consistent with, and incorporates currently used guidelines and has the capacity to evolve as new guidelines are published. The pathway was developed to accommodate all people who may present with spine-related symptoms or concerns, is not limited to a specific symptom (i.e., pain) or spinal region (low back or neck) or chronicity and does not require a pathological diagnosis for axial spinal pain. This pathway incorporates psychological and social factors, takes into consideration public health interventions and addresses people who present with neurological symptoms, structural pathology, serious and life-threatening systemic pathology. 
These features should facilitate the implementation of this pathway in a wide variety of health care settings.

Limitations include that not all of the $68 \mathrm{GSCI}$ experts participated in the development of the care pathway and therefore is subject to bias. Like any care pathway, its value will depend on field testing to determine whether it can be implemented in communities with different cultures and resources. The care pathway described here has not yet been tested for validity and reliability. It is also necessary to determine whether it can be easily administered and utilized by all stakeholders including patients and clinicians. Resources and the implementation of the model of care are described elsewhere $[38,41]$. The final determination of the value of this care pathway will be its acceptance by patients and clinicians with different expectations or training, the ability to implement the care pathway and the determination as to whether, when implemented, it has the desired outcomes of reduced costs and disability associated with spinal disorders.

\section{Conclusion}

This is the first international and interprofessional attempt to develop a care pathway for the management of any person presenting with spine-related symptoms or concerns that incorporate the recommendations from multiple evidence-based guidelines. The decision steps are personcentered, community-based and consistent with recommendations established by the WHO, evidence-based and limited to five steps. This care pathway will need to be field tested in different cultural and resource communities to determine its utility.

Funding The Global Spine Care Initiative and this study were funded by grants from the Skoll Foundation and NCMIC Foundation. World Spine Care provided financial management for this project. The funders had no role in study design, analysis, or preparation of this paper.

\section{Compliance with ethical standards}

Conflict of interest SH declares funding to UOIT from Skoll Foundation, NCMIC Foundation through World Spine Care. Clinical Policy Advisory Board and stock holder, Palladian Health. Advisory Board, SpineHealth.com. Book Royalties, McGraw Hill. Travel expense reimbursement-CMCC Board. CDJ is president of Brighthall Inc; she is an NCMIC Board of Director; however, neither she nor NCMIC board makes funding decisions for the NCMIC Foundation; the views in this article are those of the authors and not those of Stanford University, Stanford Health Care, or Qualcomm. RC declares funding from AHRQ to conduct systematic reviews on treatments for low back pain within last 2 years. Honoraria for speaking at numerous meetings of professional societies and non-profit groups on topics related to low back pain (no industry sponsored talks). MN declares funding from Skoll Foundation and NCMIC Foundation through World Spine Care;.
Co-Chair, World Spine Care Research Committee. Palladian Health, Clinical Policy Advisory Board member. Book Royalties Wolters Kluwer and Springer. Honoraria for speaking at research method courses. PC is funded by a Canada Research Chair in Disability Prevention and Rehabilitation at the University of Ontario Institute of Technology, and declares funding to UOIT from Skoll Foundation, NCMIC Foundation through World Spine Care. Canadian Institutes of Health Research Canada. Research Chair Ontario Ministry of Finance. Financial Services Commission of Ontario. Ontario Trillium Foundation, ELIB Mitac. Fond de Recherche and Sante du Quebec. EH declares he is a consultant for: RAND Corporation; EBSCO Information Services; Southern California University of Health Sciences; Western University of Health Sciences Data and Safety Monitoring Committee. Chair, Palmer Center for Chiropractic Research. Research Committee Cochair, World Spine Care. BNG receives speaker fees and travel reimbursement from NCMIC Speakers' Bureau; he is secretary of Brighthall Inc; the views in this article are those of the authors and not those of Stanford University, Stanford Health Care, or Qualcomm. EAc declares grants: Depuy Synthes Spine, Medtronic; Speaker's Bureau: AOSpine, Zimmer Biomet. DKG declares travel expenses: CMCC to present at the WSC Spine Conference in Botswana. OB declares he is a consultant for: Pacira Pharmaceuticals, Inc. Palladian Health. Travel expenses: World Spine Care. Stipend: World Spine Care. PB declares contribution to salary for Global Musculoskeletal Alliance (G-MUSC), The Bone and Joint Decade work. JMC declares Government Grant ESPY 1281/15. CG declares travel expenses: Palmer College to GSCI meetings. Consultant: American Chiropractic Association, Spine IQ, Healthwise, Quality Insights of Pennsylvania, RAND Co.; Prezacor, Inc. (Stock Options). PCORI (Board Member). Grants: Collaborative Care for Veterans with Spine pain and Mental Health Issues. NIH/ Kiernan Chiropractic Care in Rehabilitation at Crotched Mountain: Crotched Mountain Private Sector Integrated Chiropractic Study N/A. NCMIC Foundation Chiropractic services, Assessment of Chiropractic Treatment for Low Back Pain; RAND Subcontract, Department of Defense Prime Award \#W81XWH-11-2-017 Sub \#9920110071. MH declares travel support from World Spine Care. JM declares general research resources from USF Research Center. Research grants from funding agencies: FEMA, US Department of Homeland Security (EMW-2013-FP-00723). Palladian Health Advisory board: Clinical Policy and Advisory Board,. Intellectual property rights: Inventor of Web-based system to deliver exercise (Employer - USF: copyright holder). TM declares Fellowship grant-Medtronics. JM declares WSC Board Member. EM declares AO Spine Africa Faculty courses - honorarium. GO declares he is a consultant and receives travel support as Clinic Director, World Spine Care. KR declares funding to UOIT from Skoll Foundation, NCMIC Foundation through World Spine Care. HR declares funding to UOIT from Skoll Foundation, NCMIC Foundation through World Spine Care. ES declares funding from Başkent University Research Fund. LV declares funding to UOIT from Skoll Foundation and NCMIC Foundation through World Spine Care. WW declares Palladian Health, Clinical Policy Advisory Board member. HY declares funding to UOIT from Skoll Foundation, NCMIC Foundation through World Spine Care, the remaining authors declare that they have no conflict of interest.

Open Access This article is distributed under the terms of the Creative Commons Attribution 4.0 International License (http://creativeco mmons.org/licenses/by/4.0/), which permits unrestricted use, distribution, and reproduction in any medium, provided you give appropriate credit to the original author(s) and the source, provide a link to the Creative Commons license, and indicate if changes were made. 


\section{References}

1. Hurwitz EL, Randhawa K, Yu H, Cote P, Haldeman S (2018) The Global Spine Care Initiative: a summary of the global burden of low back and neck pain studies. Eur Spine J. https://doi. org/10.1007/s00586-017-5432-9

2. Hurwitz EL, Randhawa K, Torres P, Yu H, Verville L, Hartvigsen J, et al (2017) The Global Spine Care Initiative: a systematic review of individual and community-based burden of spinal disorders in rural populations in low- and middle-income communities. Eur Spine J. https://doi.org/10.1007/s00586-017-5393-z

3. Martin BI, Deyo RA, Mirza SK, Turner JA, Comstock BA, Hollingworth W et al (2008) Expenditures and health status among adults with back and neck problems. JAMA 299(6):656-664

4. Dieleman JL, Baral R, Birger M, Bui AL, Bulchis A, Chapin A et al (2016) US spending on personal health care and public health, 1996-2013. JAMA 316(24):2627-2646

5. Hartvigsen J, Hancock MJ, Kongsted A, Louw Q, Ferreira ML, Genevay $\mathrm{S}$ et al (2018) What low back pain is and why we need to pay attention. Lancet 391(10137):2356-2367

6. Buchbinder R, van Tulder M, Öberg B, Costa LM, Woolf A, Schoene M et al (2018) Low back pain: a call for action. Lancet 391(10137):2384-2388

7. Bishop PB, Wing PC (2003) Compliance with clinical practice guidelines in family physicians managing worker's compensation board patients with acute lower back pain. Spine J 3(6):442-450

8. Bishop PB, Wing PC (2006) Knowledge transfer in family physicians managing patients with acute low back pain: a prospective randomized control trial. Spine J 6(3):282-288

9. Briggs AM, Towler SC, Speerin R, March LM (2014) Models of care for musculoskeletal health in Australia: now more than ever to drive evidence into health policy and practice. Aust Health Rev 38(4):401-405

10. Joshi R, Alim M, Kengne AP, Jan S, Maulik PK, Peiris D et al (2014) Task shifting for non-communicable disease management in low and middle income countries-a systematic review. PLoS ONE 9(8):e103754

11. Mills A (2014) Health care systems in low-and middle-income countries. N Engl J Med 370(6):552-557

12. Veillard J, Cowling K, Bitton A, Ratcliffe H, Kimball M, Barkley $S$ et al (2017) Better measurement for performance improvement in low-and middle-income countries: the Primary Health Care Performance Initiative (PHCPI) experience of conceptual framework development and indicator selection. Milbank Q 95(4):836-883

13. Abegunde DO, Mathers CD, Adam T, Ortegon M, Strong K (2007) The burden and costs of chronic diseases in low-income and middle-income countries. The Lancet 370(9603):1929-1938

14. Beveridge M, Howard A (2004) The burden of orthopaedic disease in developing countries. J Bone Joint Surg Am 86-A(8):1819-1822

15. Bitton A, Ratcliffe HL, Veillard JH, Kress DH, Barkley S, Kimball $M$ et al (2017) Primary health care as a foundation for strengthening health systems in low-and middle-income countries. J Gen Intern Med 32(5):566-571

16. Cherkin DC, Deyo RA, Wheeler K, Ciol MA (1994) Physician variation in diagnostic testing for low back pain. Who you see is what you get. Arthritis Rheumatol 37(1):15-22

17. Schrijvers GHA, Huiskes N (2012) The care pathway: concepts and theories: an introduction. Int J Integr Care 12:e192

18. Chawla A, Westrich K, Matter S, Kaltenboeck A, Dubois R (2016) Care pathways in US healthcare settings: current successes and limitations, and future challenges. Am J Manag Care 22(1):53-62

19. Lawal AK, Rotter T, Kinsman L, Machotta A, Ronellenfitsch U, Scott SD et al (2016) What is a clinical pathway? Refinement of an operational definition to identify clinical pathway studies for a Cochrane systematic review. BMC Med 14(1):35

20. Cavlan O, Dash P, Drouin J, Fountaine T, Riahi F (2011) Using care pathways to improve health systems. Health Int 11:6-17

21. Fourney DR, Dettori JR, Hall H, Härtl R, McGirt MJ, Daubs MD (2011) A systematic review of clinical pathways for lower back pain and introduction of the Saskatchewan Spine Pathway. Spine 36:S164-S171

22. Murray CJ, Richards MA, Newton JN, Fenton KA, Anderson HR, Atkinson C et al (2013) UK health performance: findings of the Global Burden of Disease Study 2010. The Lancet 381(9871):997-1020

23. Johnson CD, Haldeman S, Nordin M, Chou R, Côté P, Hurwitz EL, et al The Global Spine Care Initiative: methodology, contributors, and disclosures. Eur Spine J. https://doi.org/10.1007/s0058 6-018-5723-9

24. Acaroglu E, Mmopelwa T, Yuksel S, Ayhan S, Nordin M, Randhawa K, et al (2017) The Global Spine Care Initiative: a consensus process to develop and validate a stratification scheme for surgical care of spinal disorders as a guide for improved resource utilization in low- and middle-income communities. Eur Spine J. https ://doi.org/10.1007/s00586-017-5332-z

25. Acaroglu E, Nordin M, Randhawa K, Chou R, Cote P, Mmopelwa $\mathrm{T}$, et al (2018) The Global Spine Care Initiative: a summary of guidelines on invasive interventions for the management of persistent and disabling spinal pain in low- and middle-income communities. Eur Spine J. https://doi.org/10.1007/s00586-017-5392-0

26. Ameis A, Randhawa K, Yu H, Cote P, Haldeman S, Chou R, et al (2017) The Global Spine Care Initiative: a review of reviews and recommendations for the non-invasive management of acute osteoporotic vertebral compression fracture pain in low- and middle-income communities. Eur Spine J. https://doi.org/10.1007/ s00586-017-5273-6

27. Cedraschi C, Nordin M, Haldeman S, Randhawa K, KopanskyGiles D, Johnson CD, et al (2018) The Global Spine Care Initiative: a narrative review of psychological and social issues in back pain in low- and middle-income communities. Eur Spine J. https ://doi.org/10.1007/s00586-017-5434-7

28. Chou R, Cote P, Randhawa K, Torres P, Yu H, Nordin M, et al (2018) The Global Spine Care Initiative: applying evidence-based guidelines on the non-invasive management of back and neck pain to low- and middle-income communities. Eur Spine J. https://doi. org/10.1007/s00586-017-5433-8

29. Green BN, Johnson CD, Haldeman S, Kane EJ, Clay MB, Griffith E, et al The Global Spine Care Initiative: public health and prevention interventions for common spine disorders in low- and middle-income communities. Eur Spine J. https://doi.org/10.1007/ s00586-018-5635-8

30. Haldeman S, Johnson CD, Chou R, Nordin M, Côté P, Hurwitz EL et al The Global Spine Care Initiative: classification system for spine-related concerns. Eur Spine J. https://doi.org/10.1007/ s00586-018-5724-8

31. Nordin M, Randhawa K, Torres P, Yu H, Haldeman S, Brady O, et al (2018) The Global Spine Care Initiative: a systematic review for the assessment of spine-related complaints in populations with limited resources and in low- and middle-income communities. Eur Spine J. https://doi.org/10.1007/s00586-017-5446-3

32. Green BN, Johnson CD, Haldeman S, Griffith E, Clay MB, Kane EJ et al (2018) A scoping review of biopsychosocial risk factors and co-morbidities for common spinal disorders. PLoS ONE 13(6): $\mathrm{e} 0197987$

33. Kamper SJ, Apeldoorn A, Chiarotto A, Smeets R, Ostelo R, Guzman J et al. (2015) Multidisciplinary biopsychosocial rehabilitation for chronic low back pain: Cochrane systematic review and meta-analysis. BMJ 350:h444 
34. Qaseem A, Wilt TJ, McLean RM, Forciea MA, Clinical Guidelines Committee of the American College of P (2017) noninvasive treatments for acute, subacute, and chronic low back pain: a clinical practice guideline from the American College of Physicians. Ann Intern Med. 166(7):514-530

35. World Health Organization (2010) Framework for action on interprofessional education and collaborative practice. World Health Organization, Geneva. http://www.who.int/hrh/resources/frame work_action/en/

36. Organization WH (2016) Framework on integrated, peoplecentred health services. Report by the Secretariat, p 18. WHO, Geneva. www.who.int/servicedeliverysafety/areas/people-centr ed-care/en/on

37. Organization WH (2013) Transforming and scaling up health professionals' education and training: World Health Organization guidelines 2013. World Health Organization

38. Johnson CD, Haldeman S, Chou R, Nordin M, Green BN, Côté P, Hurwitz EL et al The Global Spine Care Initiative: model of care and implementation Eur Spine J. https://doi.org/10.1007/s0058 6-018-5720-Z

39. Hoy D, Geere JA, Davatchi F, Meggitt B, Barrero LH (2014) A time for action: Opportunities for preventing the growing burden and disability from musculoskeletal conditions in lowand middle-income countries. Best Pract Res Clin Rheumatol 28(3):377-393

40. Foster NE, Anema JR, Cherkin D, Chou R, Cohen SP, Gross DP et al (2018) Prevention and treatment of low back pain: evidence, challenges, and promising directions. Lancet 391(10137):2368-2383

41. Kopansky-Giles D, Johnson CD, Haldeman S, Chou R, Côté $\mathrm{P}$, Green BN, Nordin $\mathrm{M}$ et al The Global Spine Care Initiative: resources to implement a spine care program. Eur Spine J. https ://doi.org/10.1007/s00586-018-5725-7

\section{Affiliations}

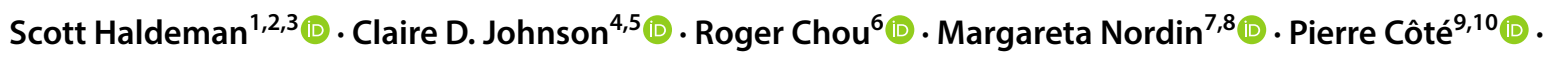

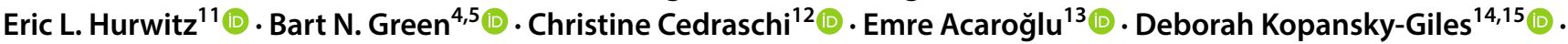

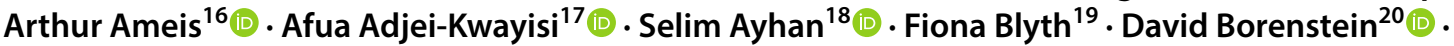
O'Dane Brady ${ }^{21}$ (1) Peter Brooks ${ }^{22}$. Connie Camilleri ${ }^{23}$. Juan M. Castellote ${ }^{24}$. Michael B. Clay ${ }^{25}$. Fereydoun Davatchi ${ }^{26}$ (D) . Robert Dunn ${ }^{27}$ (D) Christine Goertz ${ }^{28,29}$ (C) . Erin A. Griffith ${ }^{30} \cdot$ Maria Hondras $^{31}$ (D) Edward J. Kane ${ }^{32}$ (1) Nadège Lemeunier ${ }^{33} \cdot$ John Mayer $^{34} \cdot$ Tiro Mmopelwa $^{35} \cdot$ Michael Modic $^{36}$ - Jean Moss ${ }^{37}$. Rajani Mullerpatan ${ }^{38}$. Elijah Muteti ${ }^{39} \cdot$ Lillian Mwaniki $^{40} \cdot$ Madeleine Ngandeu-Singwe $^{41} \cdot$ Geoff Outerbridge $^{42}(1)$. Kristi Randhawa ${ }^{9,10}$ - Heather Shearer ${ }^{10} \cdot$ Erkin Sönmez $^{43}$ (1) $\cdot{\text { Carlos } \text { Torres }^{44} \text {. Paola Torres }}^{45} \cdot$ Leslie Verville $^{9,10}$. Adriaan Vlok ${ }^{46} \cdot$ William Watters III ${ }^{47,48} \cdot$ Chung Chek Wong ${ }^{49} \cdot$ Hainan $\mathrm{Yu}^{9,10}$

Claire D. Johnson

globalspinecareinitiative@gmail.com

1 Department of Epidemiology, School of Public Health, University of California Los Angeles, Los Angeles, CA, USA

2 Department of Neurology, University of California, Irvine, Irvine, CA, USA

3 World Spine Care, Santa Ana, CA, USA

4 National University of Health Sciences, Lombard, IL, USA

5 Qualcomm Health Center, Stanford Health Care, San Diego, CA, USA

6 Department of Medical Informatics and Clinical Epidemiology and Department of Medicine, Oregon Health \& Science University, Portland, OR, USA

7 Departments of Orthopedic Surgery and Environmental Medicine, New York University, New York, NY, USA

8 World Spine Care Europe, Holmfirth, UK

9 Faculty of Health Sciences, University of Ontario Institute of Technology, Oshawa, Canada

10 UOIT-CMCC Centre for Disability Prevention and Rehabilitation, Toronto, Canada

11 Office of Public Health Studies, University of Hawai'i, Mānoa, Honolulu, HI, USA
12 Division of General Medical Rehabilitation and Division of Clinical Pharmacology and Toxicology, Multidisciplinary Pain Centre, Geneva University Hospitals, Geneva, Switzerland

13 ARTES Spine Center, Ankara, Turkey

14 Department of Research, Canadian Memorial Chiropractic College, North York, ON, Canada

15 Department of Family and Community Medicine, University of Toronto, Toronto, ON, Canada

16 Faculty of Medicine Certification Program in Insurance Medicine and MedicoLegal Expertise, University of Montreal, Toronto, ON, Canada

17 Ridge Regional Hospital, Ghana World Spine Care, Accra, Greater Accra, Ghana

18 ARTES Spine Center, Acibadem University, Ankara, Turkey

19 Concord Clinical School, University of Sydney, Concord, NSW, Australia

20 Arthritis and Rheumatism Associates, The George Washington University Medical Center, Potomac, MD, USA

21 World Spine Care, Tampa, FL, USA

22 Centre for Health Policy, School of Population and Global Health, University of Melbourne, Toorak, Melbourne, VIC, Australia 
23 St. Michael's Hospital, North York, ON, Canada

24 National School of Occupational Medicine, Carlos III Institute of Health and Physical Medicine and Rehabilitation, University of Complutense, Madrid, Madrid, Spain

25 Rehabilitation Care Line, Physical Medicine and Rehabilitation, Cincinnati Veterans Affairs Medical Center, Cincinnati, OH, USA

26 Rheumatology Research Center, Tehran University of Medical Sciences, Tehran, Tehran, Iran

27 Department of Orthopaedic Surgery, University of Cape Town and Groote Schuur Hospital, Cape Town, Western Cape, South Africa

28 Palmer College of Chiropractic, Davenport, IA, USA

29 The Spine Institute for Quality, Davenport, IA, USA

30

31 Department of Anesthesiology, University of Kansas, Medical Center, Kansas City, KS, USA

32 College of Rehabilitative Sciences, Doctor of Physical Therapy Program, University of St. Augustine for Health Sciences, San Marcos, CA, USA

33 Institut Franco-Européen de Chiropraxie, Toulouse, France

34 U.S. Spine \& Sport Foundation, San Diego, CA, USA

35 ARTES Ankara Spine Centre, Life Gaborone Hospital, Gaborone, Botswana

36 Cleveland Clinic, Lerner College of Medicine, Cleveland, $\mathrm{OH}, \mathrm{USA}$
37 Canadian Memorial Chiropractic College, President Emerita, Toronto, ON, Canada

38 Mahatma Gandhi Mission Institute of Health Sciences, MGM School of Physiotherapy, Navi Mumbai, Maharashtra, India

39 Moi University/Moi Teaching \& Referral Hospital, Eldoret, Kenya

40 Law Society of Kenya, Nairobi, Kenya

41 Faculty of Medicine and Biomedical Sciences, The University of Yaoundé I, Yaoundé, Center Region, Cameroon

42 World Spine Care and Canadian Memorial Chiropractic College, Chelsea, QC, Canada

43 Department of Neurological Surgery, Başkent University School of Medicine, Ankara, Turkey

44 Department of Medical Imaging, The Ottawa Hospital, University of Ottawa, Ottawa, ON, Canada

45 Exercise Science Laboratory, School of Kinesiology, Faculty of Medicine, Universidad Finis Terrae, Santiago, Chile

46 Division of Neurosurgery, University of Stellenbosch, Bellville, Western Cape, South Africa

47 Department of Orthopedic Weill Cornell Medical School and Institute of Academic Medicine, Houston Methodist Hospital, Houston, TX, USA

48 Department of Orthopedic Surgery, University of Texas Medical Branch, Galveston, TX, USA

49 Sarawak General Hospital, Kuching, Sarawak, Malaysia 